\title{
Review
}

\section{Aquatic Therapy for Persons with Neuromuscular Diseases - A Scoping Review}

\author{
Anna Ogonowska-Slodownik ${ }^{\mathrm{a}, *}$, Ana Angélica R. de Lima ${ }^{\mathrm{b}}$, Luciana Cordeiro $^{\mathrm{c}}$, Natalia \\ Morgulec-Adamowicz ${ }^{\mathrm{a}}$, María Alonso-Fraile ${ }^{\mathrm{d}}$ and Javier Güeita-Rodríguez ${ }^{\mathrm{e}}$ \\ ${ }^{a}$ Faculty of Rehabilitation, Jozef Pilsudski University of Physical Education in Warsaw, Warsaw, Poland \\ ${ }^{\mathrm{b}}$ School of Physical Education and Sport, University of São Paulo, São Paulo, Brazil \\ ${ }^{\mathrm{c}}$ Occupational Therapy Graduation Course, Faculty of Medicine, Federal University of Pelotas, Pelotas, Brazil \\ ${ }^{\mathrm{d}}$ Aquatic Therapy Unit, San José Institute Foundation, Madrid, Spain \\ ${ }^{\mathrm{e}}$ Department of Physical Therapy, Occupational Therapy, Physical Medicine and Rehabilitation, Research Group \\ of Humanities and Qualitative Research in Health Science of Universidad Rey Juan Carlos (Hum\&QRinHS), \\ Madrid, Spain
}

Pre-press 10 January 2022

\begin{abstract}
.
Background: Aquatic exercise is among the most common physical activity modalities performed by people with disabilities. Objective: The present paper reviews currently-available research on aquatic therapy (AT) for persons with neuromuscular diseases (NMD).

Methods: A scoping review of the existing literature was conducted on PubMed, Embase, Medline, Scopus, Web of Science, SPORTdiscus, CINAHL and Lilacs from the earliest date available until October 2020. It follows the methodological framework for conducting a scoping review proposed by the Joanna Briggs Institute.

Results: A total of 28 articles were analyzed and the study parameters grouped by the topography of NMD; most of the studies $(n=16)$ addressed myopathies. A considerable increase in the number of studies was found over time, and heterogeneity was identified across and within AT interventions for persons with NMD; hence, to allow more effective interpretation of study results, there is a need to standardize the fundamental parameters and procedures for AT.

Conclusions: This scoping review provides a comprehensive outline of available literature; the findings could serve as a starting point for clinical studies on the effects of AT on persons with NMD, and encourage a more coherent approach to their design.
\end{abstract}

Keywords: Hydrotherapy, muscular dystrophy, myopathy, scoping review, neuromuscular diseases

\section{INTRODUCTION}

${ }^{*}$ Correspondence to: Anna Ogonowska-Slodownik, Josef Pilsudski University of Physical Education in Warsaw, Faculty of Rehabilitation, ul. Marymoncka 34, 00-968 Warsaw, Poland. Tel./Fax: +4822835 47 98; E-mail: anna.ogonowskaslodownik@ gmail.com.
Neuromuscular diseases (NMD) comprise a wide group of progressive, acquired or hereditary diseases that affect the peripheral nervous system, anterior horn cells, nerve roots, plexi, peripheral nerves, neuromuscular junction and/or muscles [1-4]. The onset 
of symptoms may occur in childhood or adulthood, and life expectancy depends on the type of disorder and the severity of its evolution [2,5]. Each type of NMD has a heterogeneous and complex nature affecting multiple body systems [4]. Structural and functional changes arise with the progression of the disease [6-9], most of which affect functional mobility, balance [10] and respiratory function [11]. Some NMDs are characterized by a progressive decline in respiratory function; this results in a cardiopulmonary intolerance to exercise that impairs activities of daily living (ADL) and social participation among patients [12].

The guidelines developed by NMD care groups recommend the use of low-impact exercises [13] that can be performed during aquatic therapy (AT) [6-9]. These exercises combine strengthening activities and aerobic exercise to maintain structure and function $[14,15]$. Currently, such aquatic exercise is among the most common physical activities chosen by children with neuromotor disorders [16, 17] and can be used in all phases of disease progression. The aquatic environment provides a series of hydrostatic and hydrodynamic characteristics [18], which make exercise feasible for persons with NMD, as when not supported by the water, they can no longer perform movements against gravity. The buoyancy provided by the water provides support and unloads the joints, allowing persons with NMD to move freely while performing task-specific exercises associated with daily life; should they perform such movements out of the water, they could experience significant pain or problems in physical functioning [19]. In addition, the hydrodynamic resistance ensures the use of multi-directional strengthening exercises to improve cardiovascular health [18]. Furthermore, the aquatic setting helps people to maintain gross motor function [20], allows neuropathic patients to improve their balance and gait in without risk of falling or pain [21], and offers an enriched environment that motivates participation, optimizing the functioning of persons with NMD [22].

However, although AT has demonstrated clear benefits in treating other neurological pathologies of the central nervous system [23-25], its application is less common among persons with NMD. This may be due to the lack of validated protocols, the comparatively small number of professionals with proper training, and the various accessibility problems associated with the technique [22].

The objective of a scoping review is to assess the extent of a body of literature on a particular topic, with a key goal being to examine emerging evidence to ensure that further research in that area would be beneficial [26]. Furthermore, a scoping review should identify a sufficient number of studies and inform researchers as to whether a full systematic review is needed [27]. Three recent systematic reviews which assessed the effects of AT on patients with neurological problems [23-25] did not provide much information about people with NMD.

In order to provide wide coverage of the available publications, the following two research questions were formulated: What research on AT for persons with NMD is currently available, and how has AT been applied in persons with NMD? Hence, the main purpose of this study was to map the available research interventions on AT for individuals with NMD; more specifically, to describe the characteristics of the study groups and the procedures performed, and identify the outcome measurements in AT research.

\section{MATERIALS AND METHODS}

The study followed the methodological framework for conducting a scoping review proposed by the Joanna Briggs Institute [26]. This framework comprises nine stages: 1) Defining and aligning the objective/s and question/s; 2) Developing and aligning the inclusion criteria with the objective/s and question/s; 3) Describing the planned approach to the evidence search and selection, as well as data extraction and presentation of the evidence; 4) Searching for the evidence; 5) Selecting the evidence; 6) Extracting the evidence; 7) Analyzing the evidence; 8) Presenting the results; 9) Summarizing the evidence in relation to the purpose of the review, making conclusions and noting any implications of the findings. Stages 1-3 were executed during the preparation of the scoping review. Stages 4-6 are presented in the Methods section, stages 7-8 in the Results section and stage 9 in the Discussion and Conclusions. The present study was based on the PRISMA-ScR (Preferred Reporting Items for Systematic reviews and Meta-Analyses extension for Scoping Reviews) checklist [28].

\section{Searching for the evidence}

A comprehensive search was conducted in October 2020 by two independent reviewers in eight data sources: PubMed, Embase, MEDLINE, Scopus, Web of Science, SPORTdiscus, CINAHL and Lilacs. The 
Table 1

Terms Describing Neuromuscular Diseases

\begin{tabular}{ll}
\hline Structure affected & Disease (MeSH terms) \\
\hline CNS & Primary lateral sclerosis \\
Upper motor neuron and / & Amyotrophic Lateral Sclerosis \\
or lower motor neuron & Progressive bulbar pals* \\
diseases & Progressive muscular atrophy* \\
& Spinal muscular atrophy* \\
& Poliomyelitis \\
& Post-poliomyelitis Syndrome \\
\hline PNS & Neurophathy* \\
Neuropathies & Polyneuropathy* \\
& Charcot-Marie-Tooth Disease \\
& Guillain-Barre Syndrome \\
& Dejerine-Sottas Disease \\
& Disease, refsum \\
& Krabbe Disease \\
\hline Neuromuscular junction & Myasthenia Gravis \\
& Myasthenic Syndrome \\
\hline Myopathy & Botulism \\
\hline & Muscular Dystrophy* \\
& Myotonic Dystrophy* \\
\hline
\end{tabular}

CNS - central nervous system; MeSH-Medical Subject Headings; PNS - peripheral nervous system.

search used two groups of keywords. The first group described all types of NMDs with Medical Subject Headings (MeSH terms) (Table 1). The terms were connected with the Boolean search operator "OR". The second group examined the intervention, and the search comprised a combination of the following keywords: aquatic exercise OR aquatic physical therapy OR swimming OR aquatic therapy OR water exercise. There was no date limit for the search.

The reference lists of all included articles were hand-searched for new articles. The authors also checked their own libraries for additional literature.

\section{Selecting the evidence}

Study selection was conducted independently by at least two authors, who were native speakers of Spanish and fluent in English and Portuguese. The first step in study selection consisted of analyzing titles and abstracts; this step included articles written in English, Spanish and Portuguese describing active AT in persons with NMD. The following exclusion criteria were applied: animal studies; passive AT used for persons with NMD; articles describing treatments other than AT and/or with study groups other than persons with NMD; reviews or meta-analysis.

The second step was to analyze the full texts of the potentially relevant papers. The studies eligible for inclusion must describe the content of the intervention: who received it, where it occurred, and how it was delivered. The following were excluded: any articles which presented passive AT, any articles without intervention, or any articles that were not original, lacked full text or were not in English, Spanish or Portuguese. Inclusion of each paper was based on the assessment from two independent reviewers and full agreement was required. In the case of disagreement, consensus was reached by consulting a third reviewer.

\section{Extracting the evidence}

The following descriptive data were extracted: the name of the first author, year of publication, study design, primary research question, sample size, age and gender of the participants, diagnosis, intervention description, measuring instrument and outcome, water temperature. The tables were organized based on the participants' diagnosis. Data extraction for the included studies was divided between six authors according to their ability in the language of the article.

\section{RESULTS}

The database search revealed 2171 records, and another 15 were found through other sources. A flow diagram of the identified studies is presented in Fig. 1. After excluding duplicates using EndNote 20 software, 1479 records were screened based on the title and abstract. Full-text versions of 89 articles were assessed for eligibility. Finally, 31 articles met the inclusion criteria. Two studies presented the same data $[29,30]$; in this case, the one with less information was excluded [30]. Another two studies did not present enough information about AT [31, 32], more specifically with regard to inclusion criteria of the second step of the study selection, and these were also excluded. The final analysis therefore included data from 28 articles. All included articles had been published in peer-reviewed journals. Eleven of the included articles were published in Portuguese with an English abstract. Out of 28 studies, 18 (64\%) had been published after 2010 .

Regarding the type of study, 19 were descriptive case studies [29, 33-50], four were quasiexperimental studies [19, 51-53], two were randomized clinical trials $[20,21]$, one was a longitudinal prospective study [54], one was a longitudinal retrospective study [55] and one was a cross-sectional study [56]. The outcomes of analyzed studies were grouped into the following general categories (Table 2): functional performance (walking, balance, activities of daily living), physiological domain 


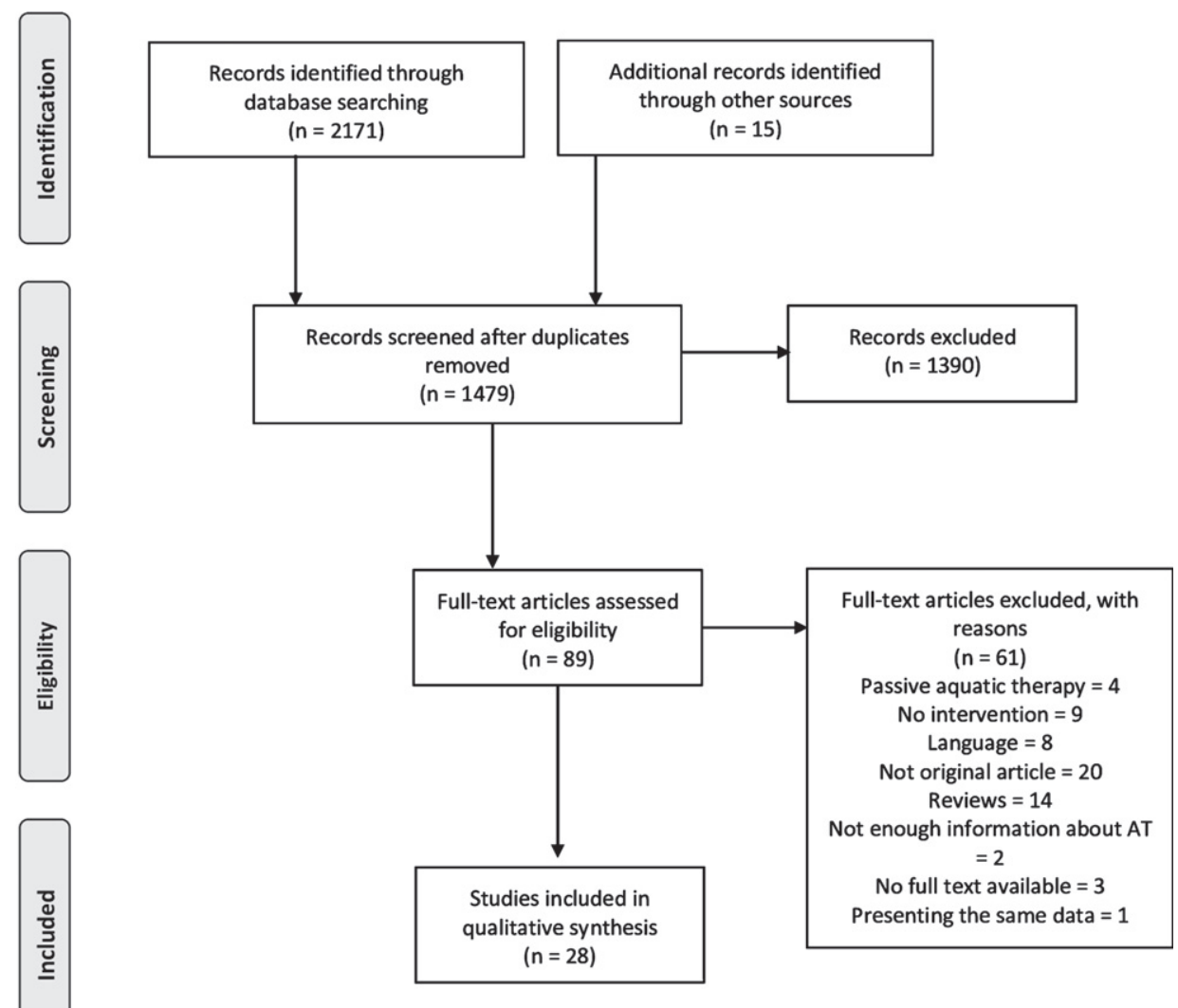

Fig. 1. Flow Diagram of Identified Publications.

(cardiorespiratory, strength and somatic aspects), psychosocial domain (quality of life, pain, physical, psychological, mental, emotional and social aspects) and aquatic skills.

Regarding the topography of NMD, 16 studies addressed myopathies, eight examined central nervous system (CNS) diseases, three described the use of AT for peripheral nervous system (PNS) diseases and one study reported sample including various NMDs [54]. No studies reporting the use of AT for neuromuscular junction disorders.

\section{Myopathy}

Sixteen studies included persons with myopathy (Table 3), including eleven on Duchenne Muscular Dystrophy (DMD), [20, 29, 35, 37, 44, 46, 49, 52, $53,55,56]$ three on Limb-girdle Muscular Dystrophy (LGMD) [36, 38, 56] and one on congenital Muscular Dystrophy (MD) [48]; two studies did not report the type of MD [33,47]. Overall, the six- teen studies represented a total of 122 participants, of whom $>95 \%$ were male with DMD $(n=114)$ and MD $(n=4)$, and about $5 \%$ were female with LGMD and MD; the age ranged from two to thirty-five years old. Six studies used a standardized assessment of the Egen Klassification Scale (EK), the North Star Ambulatory Assessment, the Motor Function Measurement and the Vignos Scale, either to characterize the participants or as an outcome measure.

The mean duration of the AT intervention was about 29 weeks ( $M=29.23, \mathrm{SD}=30.5)$; however, the programs ranged in length from 5 to 96 weeks. The length of individual AT sessions ranged from 30 to $60 \mathrm{~min}$. The water temperature was described in nine of sixteen studies, and ranged from 28.8 to $36^{\circ} \mathrm{C}$, with most of the studies above $32^{\circ} \mathrm{C}$. In the majority of the studies $(n=13)$ the frequency of AT varied from one to three days per week.

The most commonly-analyzed outcomes were from the physiological domain, described in eleven studies, and functional performance, reported in 
Table 2

Measurements Under General Outcome Categories

\begin{tabular}{|c|c|c|c|}
\hline Functional performance & Physiological domain & Psychosocial domain & Aquatic skills \\
\hline 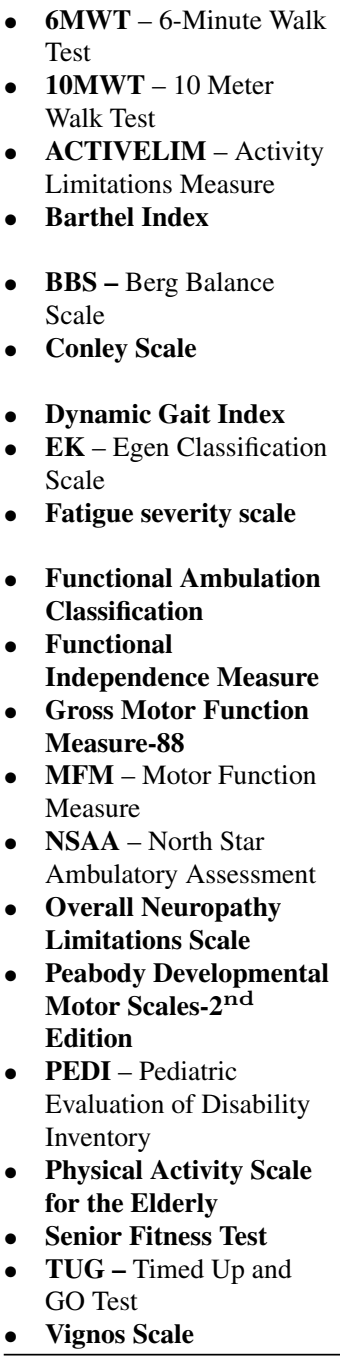 & 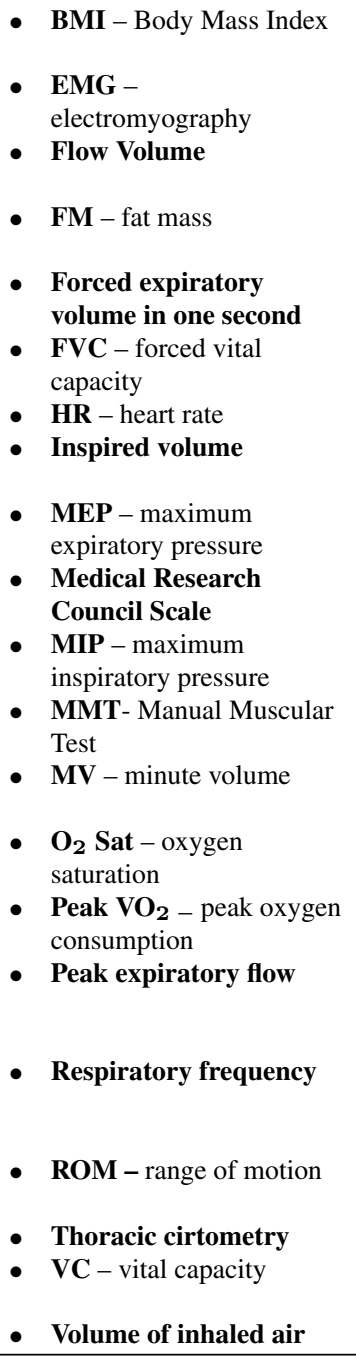 & $\begin{array}{ll}\text { - } & \text { Beck Anxiety Inventory } \\
\text { - } & \text { Carer quality of life } \\
\text { questionnaire } \\
\text { - Neuropathic Pain Scale } \\
\text { - Pediatric quality of life } \\
\text { inventory } \\
\text { - Self-Perception Profile for } \\
\text { Adolescents } \\
\text { - SF36 - Short Form (36) } \\
\text { - Health Survey } \\
\text { - VAS - Visual Analog Scale } \\
\text { - Inild Health Utility 9D } \\
\text { - Child Stress Symptoms } \\
\text { Inventory }\end{array}$ & $\begin{array}{ll}- & \text { WOTA 1 - Water } \\
\text { Orientation Test Alyn 1 } \\
\text { - } \\
\text { Aquatic skills evaluation } \\
\text { (Likert scale) }\end{array}$ \\
\hline
\end{tabular}

eight studies. The outcomes from the physiological domain often focused on respiratory function $(n=8)$ and motor function $(n=9)$. In two studies [36, 49] forced vital capacity in persons with DMD and LGMD did not change after AT intervention, while in other two $[20,53]$ a decrease was reported. The maximum inspiratory pressure increased in Caromano et al. [56] and Silva et al. [49], but decreased in Ramos et al. [52]. Maximum expiratory pressure decreased in Caromano et al. [56] and Ramos et al. [52] but was maintained in Silva et al. [49]. No change in peak expiratory flow was reported by Ramos et al. [52], but a decrease was observed in two other studies $[49,53]$.
In some studies related to AT interventions for individuals with myopathy, functional performance was measured by validated and reliable instruments. The EK score was analyzed in persons with DMD. Honório et al. [29] and Ferreira et al. [55] found EK to improve after long-term AT (89-104 weeks); however, no such change was reported by Silva et al. [49] after 10 sessions.

The quality of life was reported in three studies $[20,35,36]$ and aquatic skills were evaluated in three. Ferreira et al. [55] applied the Likert Scale based evaluation on Halliwick Method, Israel [38] developed her own instrument, and DiBiasio et al. [36] described percentage system of aquatic skills progression. 
Table 3

Study Characteristics - Research on Myopathy

\begin{tabular}{|c|c|c|c|c|c|c|c|}
\hline Reference & Study aim & Sample & Intervention program & Exercise parameters & $\begin{array}{l}\text { Water tem- } \\
\text { perature }\end{array}$ & Measuring instrument and outcome & $\begin{array}{l}\text { Study } \\
\text { design }\end{array}$ \\
\hline $\begin{array}{l}\text { Adams \& } \\
\text { Chandler } \\
\text { (1974) [33] }\end{array}$ & NR & $\begin{array}{l}\text { MD; Type: NR } \\
n=3(\mathrm{M}) \\
\text { Case } 1: 11 \text { years } \\
\text { Case } 2: 13 \text { years } \\
\text { Case } 3: 15 \text { years } \\
\text { Functional Status: } \\
\text { level } 6\end{array}$ & $\begin{array}{l}\text { AT (extended breath-holding, } \\
\text { blowing water games, } \\
\text { bobbing) + positive pressure } \\
\text { therapy (breathing and increasing } \\
\text { chest wall method) }\end{array}$ & $\begin{array}{l}\text { Phase 1: AT ( } 30 \text { min, } \\
3 \text { days/week for } 4 \\
\text { months) + positive } \\
\text { pressure therapy (1 } \\
\text { day/week for } 4 \\
\text { months) } \\
\text { Phase 2: AT ( } 30 \text { min, } \\
3 \text { days/week, duration } \\
\text { NR) + positive } \\
\text { pressure therapy (2 } \\
\text { days/week, duration } \\
\text { NR) } \\
\text { Phase 3: AT ( } 20 \text { min, } \\
4 \text { days/week for } 5 \\
\text { weeks) + positive } \\
\text { pressure therapy (NR) }\end{array}$ & NR & $\begin{array}{l}\text { Pre/post phase: } \\
\text { Phase } 1 \\
\text { VC: Case 1: } \uparrow 8 \% \text {; Case } 2: \uparrow 3 \% \text {; Case } 3 \text { : } \\
\uparrow 4 \% \\
\text { Phase } 2 \\
\text { VC: Case 1: } \uparrow 14 \% \text {; Case } 2: \text { no change; Case } \\
3: \uparrow 11 \% \\
\text { Phase } 3 \\
\text { VC: Case } 1: \uparrow 13 \% \text {; Case } 2: \uparrow 7 \% \text {; Case } 3 \text { : } \\
\uparrow 3 \%\end{array}$ & $\begin{array}{l}\text { Case } \\
\text { study }\end{array}$ \\
\hline $\begin{array}{l}\text { Atamturk \& } \\
\text { Atamturk } \\
(2018)[35]\end{array}$ & $\begin{array}{l}\text { To investigate the } \\
\text { impact of activity on } \\
\text { physical and } \\
\text { psychological health }\end{array}$ & $\begin{array}{l}\text { DMD } \\
n=1(\mathrm{M}) \\
6 \text { years }\end{array}$ & $\begin{array}{l}\text { AT (warm-up, breathing } \\
\text { exercises, water adaptation } \\
\text { activities, stretching, gross motor } \\
\text { movements, cooldown) }\end{array}$ & $\begin{array}{l}45 \mathrm{~min}, 2 \text { days/week } \\
\text { for } 8 \text { weeks }\end{array}$ & NR & $\begin{array}{l}\text { Post intervention } \\
\text { - Parents semi-structured interview: } \\
\uparrow \text { socialization and quality of life } \\
\text { slept better, got calmer and more confident }\end{array}$ & $\begin{array}{l}\text { Case } \\
\text { study }\end{array}$ \\
\hline $\begin{array}{l}\text { Caromano } \\
\text { et al. (1998) } \\
{[56]}\end{array}$ & $\begin{array}{l}\text { To study the effects of } \\
\text { physiological aspects } \\
\text { of immersion }\end{array}$ & $\begin{array}{l}\text { DMD } \\
n=20(\mathrm{M}) \\
8-15 \text { years }\end{array}$ & $\begin{array}{l}\text { AT (general active exercises, } \\
\text { walking and breathing exercises) }\end{array}$ & 40 min, 1 session & $30-32^{\circ} \mathrm{C}$ & $\begin{array}{l}\text { Pre (land, water) and post intervention } \\
\text { (land): } \\
\text { - } \mathrm{HR}: \downarrow 7.3 \mathrm{bpm} \text { (water), no change (post on } \\
\text { land) } \\
\text { - } \mathrm{MEP:} \uparrow 7.40 \mathrm{cmH}_{2} \mathrm{O} \text { (water); } \\
\downarrow 6.80 \mathrm{cmH}_{2} \mathrm{O} \text { (post on land) } \\
-\mathrm{MIP:} \downarrow 8 \mathrm{cmH}_{2} \mathrm{O} \text { (water); } \uparrow 3.80 \mathrm{cmH}_{2} \mathrm{O} \\
\text { (post on land) } \\
-\mathrm{O}_{2} \text { Sat: } \downarrow 2.7 \% \text { (water); } \uparrow 1.8 \% \text { (post on } \\
\text { land) } \\
\text { - Oral temperature: no change }\end{array}$ & $\begin{array}{l}\text { Cross- } \\
\text { sectional }\end{array}$ \\
\hline
\end{tabular}




\section{DiBiasio et al. To describe the effects LGMD (2015) [36] and viability of an $n=1(\mathrm{~F})$ individuati}

AT (walking, supine flutter kick, jumping in place in 5 feet of water, semi-prone breast stroke with a noodle, modified backstroke, active stretching, running with a floating barbell, cool down) static standing, tall kneeling, and

$45 \mathrm{~min}, 2$ days/week $\quad 28-30^{\circ} \mathrm{C} \quad \begin{aligned} & \text { Pre }(6-8 \text { weeks before intervention) and post } \\ & \text { intervention }\end{aligned}$
for 32 weeks

- BMI: $\uparrow 4.10 \mathrm{~kg} / \mathrm{cm}$

- Dynamometry: $\uparrow$ right knee flexion and extension

- FVC: no change

- Maximum ventilation volume:

$\uparrow 8 \%$

- Passive ROM: $\uparrow$ hip flexion/ extension,

right knee extension, shoulder flexion;

dorsiflexion and left knee extension

-PEDI: $\uparrow$ mobility and social function; $\downarrow$

self-care and mobility

- Self-Perception Profile for Adolescents: $\uparrow$

all areas except physical appearance

- Swimming skills: $\uparrow$

- Time spent in target HR zone:

\begin{tabular}{|c|c|c|c|c|c|c|c|}
\hline $\begin{array}{l}\text { Fachardo et al. } \\
\text { (2004) [37] }\end{array}$ & $\begin{array}{l}\text { To verify if the AT is } \\
\text { capable to delay the } \\
\text { progression of the } \\
\text { pathology }\end{array}$ & $\begin{array}{l}\text { DMD } \\
n=1(\mathrm{M}) \\
9 \text { years }\end{array}$ & $\begin{array}{l}\text { AT (stretching, strengthening of } \\
\text { the upper limbs and lower, } \\
\text { balance, trunk control, breathing } \\
\text { exercises, cool down) }\end{array}$ & $\begin{array}{l}40 \mathrm{~min}, 3 \text { days/week } \\
\text { for two phases of } 7 \\
\text { weeks }\end{array}$ & $30-32^{\circ} \mathrm{C}$ & $\begin{array}{l}\text { Pre/post phase } \\
\text { - Evaluation of activities functional } \\
\text { disorders: } \\
\text { Phase } 1: \downarrow 2 \text { pts; Phase } 2: \downarrow 1 \text { pts }\end{array}$ & $\begin{array}{l}\text { Case } \\
\text { study }\end{array}$ \\
\hline $\begin{array}{l}\text { Ferreira et al. } \\
\text { (2015) [55] }\end{array}$ & $\begin{array}{l}\text { To compare the motor } \\
\text { function on land and } \\
\text { in the water over a } \\
\text { 2-year period }\end{array}$ & $\begin{array}{l}\text { DMD } \\
n=23(\mathrm{M}) \\
15.1(4.2) \text { years }\end{array}$ & $\begin{array}{l}\text { AT (water adaptation, balance in } \\
\text { sitting and standing position, } \\
\text { exercises for trunk, upper and } \\
\text { lower limbs, rotations of } \\
\text { Halliwick Method, gait training, } \\
\text { backstroke, cool down) + PT } \\
\text { (stretching, joint mobilizations, } \\
\text { active and assisted exercises, } \\
\text { balance, standing with orthoses, } \\
\text { walking) }\end{array}$ & $\begin{array}{l}\text { AT }(40 \mathrm{~min}, 1 \\
\text { day/week for } 2 \\
\text { years }+ \text { PT ( } 50 \mathrm{~min}, 2 \\
\text { days/week for } 2 \text { years })\end{array}$ & $34^{\circ} \mathrm{C}$ & $\begin{array}{l}\text { Pre/post for each } 6 \text { months } \\
- \text { Aquatic skills evaluation (Likert scale): } \\
\uparrow 2.65 \text { pts } \\
\text { - EK: } \uparrow 0.96 \text { pts } \\
\text { - Vignos Scale: } \uparrow 0.65\end{array}$ & $\begin{array}{l}\text { Quasi- } \\
\text { experi- } \\
\text { mental }\end{array}$ \\
\hline
\end{tabular}

(Continued) 
Table 3

(Continued)

\begin{tabular}{|c|c|c|c|c|c|c|c|}
\hline Reference & Study aim & Sample & Intervention program & Exercise parameters & $\begin{array}{l}\text { Water tem- } \\
\text { perature }\end{array}$ & Measuring instrument and outcome & $\begin{array}{l}\text { Study } \\
\text { design }\end{array}$ \\
\hline $\begin{array}{l}\text { Hind et al. } \\
\text { (2017) [20] }\end{array}$ & $\begin{array}{l}\text { To assess } \\
\text { the clinical } \\
\text { effectiveness of AT in } \\
\text { maintaining physical } \\
\text { function }\end{array}$ & $\begin{array}{l}\text { DMD } \\
n=12(\mathrm{M}) \\
\text { EG: } n=8 \\
8(0.9) \text { years } \\
\text { CG: } n=4 \\
9.8(2.5) \text { years }\end{array}$ & $\begin{array}{l}\text { EG: AT (stretching, upper and } \\
\text { lower limbs exercises, balance, } \\
\text { trunk control) + self-administered } \\
\text { PT (stretching, strengthening } \\
\text { using gravity as the resistance) } \\
\text { CG: Self-administered PT } \\
\text { (stretching, strengthening using } \\
\text { gravity as the resistance) }\end{array}$ & $\begin{array}{l}\text { EG: AT ( } 30 \text { min, } 2 \\
\text { days/week for } 6 \\
\text { months) + Self- } \\
\text { administered PT ( } 4 \\
\text { days/week for } 6 \\
\text { months) } \\
\text { CG: Self-administered } \\
\text { PT (6 days/week for } 6 \\
\text { months) }\end{array}$ & $34-36^{\circ} \mathrm{C}$ & $\begin{array}{l}\text { Pre/post intervention } \\
\text { - 6MWT: EG } \downarrow 22 \mathrm{~m} \text {; CG: } \downarrow 105 \mathrm{~m} \\
\text { - Activity limitations measure: EG: } \downarrow 3.50 \\
\text { pts; CG: } \downarrow 11.67 \text { pts } \\
\text { - Carer quality of life questionnaire: EG: } \\
\uparrow 10.67 \text { pts; CG: } \uparrow 18.83 \text { pts } \\
\text { - Child Health Utility 9D Index-health } \\
\text { state utility: EG: } \uparrow 0.10 \text { pts; CG: } \uparrow 0.03 \text { pts } \\
\text { - FVC: EG: } \downarrow 7 \% \text {; CG: NR } \\
\text { - North Star Ambulatory Assessment: EG: } \\
\downarrow 2.75 \text { pts; CG: } \downarrow 5 \text { pts }\end{array}$ & $\mathrm{RCT}$ \\
\hline $\begin{array}{l}\text { Honório et al. } \\
\text { (2013) [29] }\end{array}$ & $\begin{array}{l}\text { To determine whether } \\
\text { AT is beneficial in } \\
\text { terms of functional } \\
\text { mobility }\end{array}$ & $\begin{array}{l}\text { DMD } \\
n=7(\mathrm{M}) \\
\text { AT: } n=1 \\
\text { No PA: } n=6 \\
\text { 9-11 years }\end{array}$ & AT (functional mobilisation) & $\begin{array}{l}5 \text { min, } 1-2 \\
\text { days/week for } 89 \\
\text { weeks }\end{array}$ & NR & $\begin{array}{l}\text { Five evaluations (periods NR) } \\
\text { - BMI: AT: } \downarrow 2.07 \mathrm{~kg} / \mathrm{m}^{2} \text {; no PA: } \\
\uparrow 1.91(1,27) \mathrm{kg} / \mathrm{m}^{2} \\
\text { - EK: AT: } \uparrow 8 \mathrm{pts} \text {; no PA: } \uparrow 8.83(2.78) \mathrm{pts} \\
\text { - FM: AT: } \uparrow 4.22 \% \text {; no PA: } \uparrow 4.29(2.29) \%\end{array}$ & $\begin{array}{l}\text { Case } \\
\text { study }\end{array}$ \\
\hline $\begin{array}{l}\text { Israel (2018) } \\
{[38]}\end{array}$ & $\begin{array}{l}\text { To evaluate an } \\
\text { intervention for } \\
\text { aquatic motor skills } \\
\text { learning in patients } \\
\text { with LGMD }\end{array}$ & $\begin{array}{l}\text { LGMD } \\
n=2(\mathrm{~F}) \\
\text { Case } 1: 33 \text { years } \\
\text { Case } 2: 32 \text { years }\end{array}$ & $\begin{array}{l}\text { AT (body rotations, swimming, } \\
\text { gait) }\end{array}$ & $\begin{array}{l}30 \mathrm{~min}, 1 \text { day/week } \\
\text { for } 15 \text { weeks }\end{array}$ & $33-34^{\circ} \mathrm{C}$ & $\begin{array}{l}\text { Pre/post intervention } \\
- \text { Aquatic Functional Assessment Scale: } \\
\text { Acclimation phase: Case } 1: \uparrow 1.8 \text { pts; Case } \\
2: \uparrow 1.0 \text { pts } \\
\text { Mastering of the liquid medium phase: Case } \\
1: \uparrow 1.5 \text { pts; Case } 2: \uparrow 2.8 \text { pts } \\
\text { Specific therapeutic exercises phase: Case } 1 \text { : } \\
\uparrow 0.5 \text { pts; Case } 2: \uparrow 1,7 \text { pts } \\
\text { Global body conditioning phase: Case } 1 \text { : } \\
\uparrow 2.0 \text { pts; Case } 2: \uparrow 2.5 \text { pts }\end{array}$ & $\begin{array}{l}\text { Case } \\
\text { study }\end{array}$ \\
\hline $\begin{array}{l}\text { Nunes et al. } \\
\text { (2008) [44] }\end{array}$ & $\begin{array}{l}\text { To measure changes } \\
\text { on stress in a child } \\
\text { under AT }\end{array}$ & $\begin{array}{l}\text { DMD } \\
n=1(\mathrm{M}) \\
10 \text { years }\end{array}$ & $\begin{array}{l}\text { AT (Halliwick method, Bad } \\
\text { Ragaz and hydro kinesiotherapy) }\end{array}$ & $\begin{array}{l}45 \mathrm{~min}, 2 \text { days/week } \\
\text { for } 10 \text { sessions }\end{array}$ & $33-34^{\circ} \mathrm{C}$ & $\begin{array}{l}\text { Pre/post intervention } \\
\text { - Child Stress Symptoms Inventory: } \\
\downarrow 42 \text { pts }\end{array}$ & $\begin{array}{l}\text { Case } \\
\text { study }\end{array}$ \\
\hline
\end{tabular}




\begin{tabular}{|c|c|c|c|c|c|c|c|}
\hline $\begin{array}{l}\text { Ramos et al. } \\
(2008)[52]\end{array}$ & $\begin{array}{l}\text { To evaluate the } \\
\text { respiratory muscle } \\
\text { force and peak flow in } \\
\text { patients with DMD } \\
\text { exposed to } \\
\text { non-invasive } \\
\text { ventilation and AT }\end{array}$ & $\begin{array}{l}\text { DMD } \\
n=6(\mathrm{M}) \\
\text { EG: } n=3 \\
17,6(1,2) \text { years } \\
\text { CG: } n=3 \\
13,5(0,5) \text { years }\end{array}$ & $\begin{array}{l}\text { EG: AT (passive stretching, } \\
\text { passive and active-free } \\
\text { mobilizations of knees, hips and } \\
\text { shoulders) + non-invasive } \\
\text { ventilation } \\
\text { CG: AT (passive stretching, } \\
\text { passive and active-free } \\
\text { mobilizations of knees, hips and } \\
\text { shoulders) }\end{array}$ & $\begin{array}{l}\text { EG: } 30 \text { min, } 2 \\
\text { days/week for } 20 \\
\text { sessions } \\
\text { CG: } 30 \text { min, } 2 \\
\text { days/week for } 20 \\
\text { sessions }\end{array}$ & NR & $\begin{array}{l}\text { Pre/post 10th session and post intervention } \\
\text { - MEP: EG: } \downarrow 1.4 \mathrm{cmH}_{2} 0 \text {; CG: } \uparrow 10.7 \mathrm{cmH}_{2} 0 \\
\text { - MIP: EG: } \downarrow 1.4 \mathrm{cmH}_{2} 0 \text {; CG: } \uparrow 1.3 \mathrm{cmH}_{2} 0 \\
\text { - Peak expiratory flow: EG: no change; CG: } \\
\uparrow 33,4 \mathrm{~L} / \mathrm{min}\end{array}$ & $\begin{array}{l}\text { Quasi- } \\
\text { experi- } \\
\text { mental }\end{array}$ \\
\hline $\begin{array}{l}\text { Sales et al. } \\
(2004)[46]\end{array}$ & $\begin{array}{l}\text { To verify the effects } \\
\text { of breathing exercises }\end{array}$ & $\begin{array}{l}\text { DMD } \\
n=1(\mathrm{M}) \\
6 \text { years }\end{array}$ & $\begin{array}{l}\text { AT (body segment movements, } \\
\text { breathing exercises, diving, } \\
\text { relaxation) }\end{array}$ & $\begin{array}{l}30 \mathrm{~min}, 1 \text { day/week } \\
\text { for } 6 \text { months }\end{array}$ & $34^{\circ} \mathrm{C}$ & $\begin{array}{l}\text { Six measurements (one per month) } \\
\text { - Respiratory frequency: } \downarrow 3 \mathrm{rpm} / \mathrm{min} \\
\text { - Thoracic cirtometry: } \uparrow 1.5 \mathrm{~cm} \text { in thoracic } \\
\text { perimeter during inspiration } \\
\text { - VC: no change }\end{array}$ & $\begin{array}{l}\text { Case } \\
\text { study }\end{array}$ \\
\hline $\begin{array}{l}\text { Sanders \& } \\
\text { Torres }(2010) \\
{[47]}\end{array}$ & $\begin{array}{l}\text { To analyze the effect } \\
\text { of aquatic exercise on } \\
\text { pain, fitness, and } \\
\text { independence for } \\
\text { ADL }\end{array}$ & $\begin{array}{l}\text { MD Type: NR } \\
n=1(\mathrm{~F}) \\
35 \text { years }\end{array}$ & $\begin{array}{l}\text { AT (walking, jogging, rocking, } \\
\text { kicking, jumping, and scissors) }\end{array}$ & NR & NR & $\begin{array}{l}\text { 2nd month of intervention } \\
\downarrow \text { back pain } \\
\text { 4th month of intervention } \\
\downarrow \text { back pain } \uparrow \text { arms and legs strength } \\
\text { 8th month of intervention } \\
\text { water walking distance: } 80 \text { steps, } \\
1 \text { st year of intervention } \\
\downarrow \text { back pain, } \uparrow \text { flexibility of hips and knees, } \\
\text { HR rest: } 66 \text { bpm, } \uparrow \text { independence in ADL } \\
\text { 5-year follow up } \\
\uparrow \text { body fat, } \uparrow \text { resting HR, } \downarrow \text { mobility and } \\
\text { strength, pain: no change }\end{array}$ & $\begin{array}{l}\text { Case } \\
\text { study }\end{array}$ \\
\hline $\begin{array}{l}\text { Santos et al. } \\
\text { (2016) [48] }\end{array}$ & $\begin{array}{l}\text { To verify the AT } \\
\text { interference in } \\
\text { velocity and energy } \\
\text { expenditure during } \\
\text { seated commuting on } \\
\text { a flat surface, and } \\
\text { functional reach }\end{array}$ & $\begin{array}{l}\text { MD Type: } \\
\text { Congenital } \\
n=1(\mathrm{M}) \\
6 \text { years }\end{array}$ & $\begin{array}{l}\text { AT (trunk mobilization, } \\
\text { abdominal muscle activation, } \\
\text { seated displacement training) }\end{array}$ & $\begin{array}{l}35 \mathrm{~min}, 2 \text { days/week } \\
\text { for } 12 \text { weeks }\end{array}$ & $33^{\circ} \mathrm{C}$ & $\begin{array}{l}\text { Pre/post intervention } \\
\text { - Energy expenditure index adapted for } \\
\text { sitting position: } \downarrow 252,31 \mathrm{bpm} \\
\text { - Functional Reach Test: } \uparrow 16 \mathrm{~cm} \\
\text { - EMG in immersion: } \uparrow \text { activation of bilateral } \\
\text { rectum abdominal and bilateral lumbar } \\
\text { square } \\
\text { - MFM: } \uparrow 4 ., 29 \% \\
\text { - Sitting displacement time ( } 2.0 \text { meters): } \\
\downarrow 19 \mathrm{~s}\end{array}$ & $\begin{array}{l}\text { Case } \\
\text { study }\end{array}$ \\
\hline
\end{tabular}


Table 3

(Continued)

\begin{tabular}{|c|c|c|c|c|c|c|c|}
\hline Reference & Study aim & Sample & Intervention program & Exercise parameters & $\begin{array}{l}\text { Water tem- } \\
\text { perature }\end{array}$ & Measuring instrument and outcome & $\begin{array}{l}\text { Study } \\
\text { design }\end{array}$ \\
\hline $\begin{array}{l}\text { Silva et al. } \\
\text { (2012) [49] }\end{array}$ & $\begin{array}{l}\text { To verify the impact } \\
\text { of AT on } \\
\text { non-ambulatory } \\
\text { children }\end{array}$ & $\begin{array}{l}\text { DMD } \\
n=1(\mathrm{M}) \\
12 \text { years }\end{array}$ & $\begin{array}{l}\text { AT (passive mobilization, } \\
\text { exercises to upper and lower } \\
\text { limbs and trunk flexibility; active } \\
\text { exercises for the upper and lower } \\
\text { limbs; respiratory exercises; } \\
\text { training of the function placing a } \\
\text { wheelchair inside the pool) }\end{array}$ & $60 \mathrm{~min}, 10$ sessions & $32^{\circ} \mathrm{C}$ & $\begin{array}{l}\text { Pre/post intervention } \\
\text { - EK: no change } \\
\text { - Flow Volume: } \downarrow 8 \% \\
\text { - FVC: no change } \\
\text { - MEP: no change } \\
\text { - MIP: } \uparrow 11 \% \\
\text { - Minute Volume: } \downarrow 16 \% \\
\text { - } \mathrm{O}_{2} \text { Sat: } \uparrow 3 \% \\
\text { - Peak cough flow: } \downarrow 21 \% \\
\text { - Respiratory Frequency: } \downarrow 29 \%\end{array}$ & $\begin{array}{l}\text { Case } \\
\text { study }\end{array}$ \\
\hline $\begin{array}{l}\text { Voos et al. } \\
\text { (2020) [53] }\end{array}$ & $\begin{array}{l}\text { To describe the } \\
\text { evolution of timed } \\
\text { immersion expiration } \\
\text { in patients with MD in } \\
\text { one-year follow-up }\end{array}$ & $\begin{array}{l}\text { DMD } \\
n=41(\mathrm{M}) \\
16(6.2) \text { years } \\
\text { LGMD } \\
n=16(\mathrm{NR}) \\
28(7.2) \text { years }\end{array}$ & $\begin{array}{l}\text { AT (targeted trunk, upper and } \\
\text { lower limbs control, breathing } \\
\text { exercises, throwing, catching a } \\
\text { ball, stretching, joint } \\
\text { mobilization) + PT (passive } \\
\text { stretching, exercises to recruit } \\
\text { trunk, lower and upper limbs } \\
\text { muscles, transferring from prone, } \\
\text { supine, sitting, kneeling, } \\
\text { half-kneeling, standing and } \\
\text { dynamic balance, gait) }\end{array}$ & $\begin{array}{l}\text { AT }(60 \mathrm{~min}, 2 \\
\text { days/week for } 1 \\
\text { year }+ \text { PT ( } 60 \text { min, } 2 \\
\text { days/week for } 1 \text { year) }\end{array}$ & NR & $\begin{array}{l}\text { Pre/post intervention } \\
\text { - FVC: DMD: } \downarrow 5 \% \text {; LGMD: } \downarrow 5 \% \\
\text { - MFM: DMD: } \downarrow 2 \text { pts; LGMD: } \downarrow 2 \text { pts } \\
\text { - Peak expiratory flow: } \\
\text { DMD: } \downarrow 10 \text { mL; LGMD: } \downarrow 30 \mathrm{~mL} \\
\text { - Timed immersion mouth expiration: } \\
\text { DMD: } 1 \mathrm{~s} \text {; LGMD: } \uparrow 2 \mathrm{~s} \\
\text { - Timed immersion nose expiration: } \\
\text { DMD: } 1 \text { s; LGMD: } \uparrow 5 \mathrm{~s}\end{array}$ & $\begin{array}{l}\text { Quasi- } \\
\text { experi- } \\
\text { mental }\end{array}$ \\
\hline
\end{tabular}

Inventory; PT - physiotherapy; RCT - randomized clinical trial; ROM - range of motion; VC - vital capacity. 


\section{Central nervous system diseases}

The majority of the studies in this group were conducted on persons with Post-polio syndrome (PPS), making a total of 34 people (Table 4). Most of them were women; only one study [19] included men and women. The mean age of the participants was 49.1 years old. Two studies were conducted on individuals with Spinal Muscular Atrophy (SMA) [45, 51] (51 people, 54\% men, age range $2.9-18$ years) and one case study on person with Amyotrophic Lateral Sclerosis (ALS) [39].

The duration of the single AT session ranged from 30 to 90 minutes, with the most common frequency being two days a week, as reported in five studies $[19,43,45,50,51]$. The duration of the intervention was diverse, from six weeks to two years in length, and the water temperature ranged between 27 and $34^{\circ} \mathrm{C}$, with most of the studies above $30^{\circ} \mathrm{C}$. Only two studies reported the level of immersion: one at the xiphoid process [51] and another between the waist and nipple line [46].

Two most commonly analyzed outcomes were related to functional performance and physiological domain, as described in six out of eight papers. An improvement in function and mobility was noticed in individuals with SMA and one individual with PPS, measured by the Barthel Index [50, 51]. Six studies evaluated muscle strength post AT intervention and showed improvement $[41,43,45,50,51]$ or no change [19].

Pain was assessed in three studies with the Visual Analog Scale; all reported a decrease of pain post intervention [19, 34, 50]. Three studies evaluated quality of life. In all studies, post-intervention assessment was conducted on land, following AT. Only one study [39] claimed an improvement in swimming skills, but no exact test was performed in the water.

\section{Peripheral neuropathy}

Three studies were conducted on persons with peripheral nervous system diseases: Charcot-MarieTooth Disease [40], Miller Fisher Syndrome [42] and peripheral neuropathy [21] (Table 4). This group included a total of 42 participants, of whom 52\% were female and whose age ranged from 49 to 71.8 years old.

AT interventions mainly focused on strength, balance and gait exercises and were combined with physical therapy on land. The duration of the AT session was 60 minutes, typically performed two or three times a week and the intervention lasted for four to ten weeks; the water temperature was $32-34^{\circ} \mathrm{C}$. Only one study [42] reported the level of immersion during AT to the T10 level.

All of the studies focused mainly on measuring functional performance, such as with the Berg Balance Scale; the findings either showed improvement $[21,40]$ or no change [42]. The distance of walking measured with the 6-Minute Walk Test and the gait speed measured by the Timed Up \& Go Test increased after the intervention [40, 42]. Two studies evaluated quality of life based on the Short Form-36 Health Survey [40, 42]; the results demonstrated improvement in physical function, physical, mental and general health. Only one study [21] used specific instruments for neuropathy: the Neuropathic Pain Scale and the Overall Neuropathy Limitations Scale.

\section{DISCUSSION}

The aim of this scoping review was to map the available research interventions on AT in persons with NMD. Twenty-eight publications were identified, and the review provides a full summary of the study designs, involved participants, the set-up of aquatic interventions and outcome measures utilized. Our findings indicate a considerable increase in the number of studies and a greater tendency to use validated instruments from 2010 onwards. The studies tend to focus on different aspects of the intervention outcomes such as functional performance, and the physiological and psychosocial aspects of the condition.

Most of the current research about AT in persons with NMD is based on case studies (68\%), with only two identified studies being randomized controlled trials. Therefore, it is possible that some of the inconsistent results could be due to low quality of the studies rather than the effect of AT. In addition, it can be difficult to design studies in this area as many NMDs are classified as rare diseases [57] and persons with the same disease can display a wide diversity of function, thus resulting in the creation of a heterogenous group [58]. Furthermore, persons with different types of NMD may respond very differently to exercise [59]. The researchers note that a number of studies based on various interventions designed for NMD patients are of comparatively low quality [60]. Therefore future research should include larger, well-designed trials to determine efficacy and optimal AT treatment regimens. 
Table 4

Study Characteristics - Research on Other NMDs

\begin{tabular}{|c|c|c|c|c|c|c|c|}
\hline Reference & Study aim & Sample & Intervention program & Exercise parameters & $\begin{array}{l}\text { Water tem- } \\
\text { perature }\end{array}$ & Measuring instrument and outcome & $\begin{array}{l}\text { Study } \\
\text { design }\end{array}$ \\
\hline \multicolumn{8}{|c|}{ Central Nervous System upper motor neuron and / or lower motor neuron disease } \\
\hline $\begin{array}{l}\text { Albarello \& } \\
\text { Fiorina (2012) } \\
\text { [34] }\end{array}$ & $\begin{array}{l}\text { To verify the efficacy } \\
\text { of AT in a PPS } \\
\text { individual }\end{array}$ & $\begin{array}{l}\text { PPS } \\
n=1 \\
55 \text { years }(\mathrm{F})\end{array}$ & $\begin{array}{l}\text { AT (walk, relaxation, stretching, } \\
\text { active movements for abdominal, } \\
\text { lower and upper limbs exercises, } \\
\text { scapular waist mobilization) }\end{array}$ & $\begin{array}{l}60 \mathrm{~min}, 3 \text { days/week } \\
\text { for } 20 \text { sessions }\end{array}$ & $33.5-34^{\circ} \mathrm{C}$ & $\begin{array}{l}\text { Pre/post intervention } \\
\text { - Fatigue severity scale: } \downarrow 10 \text { pts } \\
\text { - SF36: } \uparrow 23,2 \text { pts } \\
\text { - VAS - Pain scale (during activity): } \downarrow 5 \text { pts } \\
\text { - VAS - Pain scale (after rest): } \downarrow 3 \text { pts }\end{array}$ & $\begin{array}{l}\text { Case } \\
\text { study }\end{array}$ \\
\hline $\begin{array}{l}\text { Cunha et al. } \\
\text { (1996) [51] }\end{array}$ & $\begin{array}{l}\text { To evaluate the } \\
\text { potential benefits of } \\
\text { AT as a } \\
\text { supplementary } \\
\text { rehabilitation method }\end{array}$ & $\begin{array}{l}\text { SMA } \\
n=50 \\
\text { Type II: } 17(\mathrm{M}), \\
13(\mathrm{~F}) \\
\text { Mean: } 2.9 \text { years } \\
\text { Type III: } 11(\mathrm{M}), \\
9(\mathrm{~F}) \\
\text { Mean: } 18 \text { years }\end{array}$ & $\begin{array}{l}\text { AT (hydrotherapy and Halliwick } \\
\text { method) + PT (kinesiotherapy, } \\
\text { respiratory exercises and } \\
\text { stretching) }\end{array}$ & $\begin{array}{l}\text { AT ( } 30 \text { min for type II } \\
\text { and } 45 \text { min for type } \\
\text { III, } 2 \text { days/week, } 2 \\
\text { years) + PT ( } 1 \\
\text { day/week, } 2 \text { years) }\end{array}$ & $30^{\circ} \mathrm{C}$ & $\begin{array}{l}\text { Pre/post intervention } \\
\text { - Barthel Ladder: type II: } \uparrow 93 \% \text {; type III: } \\
\uparrow 100 \% \\
\text { - Degrees of deformities: } \uparrow \text { in hips, knees } \\
\text { and feet in all participants } \\
\text { - Development of scoliosis: more } \\
\text { pronounced in type II than type III } \\
\text { - MMT (only in type III): no change or } \uparrow \text { in } \\
\text { all participants } \\
\text { - Motor activities: type II - } 5 \text { participants } \\
\text { acquire sitting, } 4 \text { acquire standing, } 4 \text { lost } \\
\text { walking; type III - } 1 \text { participant acquire } \\
\text { rolling, } 1 \text { acquire sitting, } 3 \text { acquire standing, } \\
2 \text { acquire walking }\end{array}$ & $\begin{array}{l}\text { Quasi- } \\
\text { experi- } \\
\text { mental }\end{array}$ \\
\hline $\begin{array}{l}\text { Johnson } \\
\text { (1988) [39] }\end{array}$ & $\begin{array}{l}\text { To describe solution } \\
\text { to the lack of options } \\
\text { for persons disabled } \\
\text { by a chronic } \\
\text { degenerative disease }\end{array}$ & $\begin{array}{l}\text { ALS } \\
n=1(M) \\
62 \text { years }\end{array}$ & $\begin{array}{l}\text { AT (Water Exercise and } \\
\text { Therapeutic Swim - WETSwim } \\
\text { program - exercises for strength } \\
\text { and flexibility, overall } \\
\text { conditioning, recreation, and } \\
\text { socialization) }\end{array}$ & $\begin{array}{l}45 \mathrm{~min}, 1 \text { day/week } \\
\text { for NR period }\end{array}$ & $30^{\circ} \mathrm{C}$ & $\begin{array}{l}\text { - Quality of life } \uparrow \\
\text { - Performance } \uparrow \\
\text { - Swimming skills } \uparrow\end{array}$ & $\begin{array}{l}\text { Case } \\
\text { study }\end{array}$ \\
\hline $\begin{array}{l}\text { Martinez et al. } \\
\text { (2015) [41] }\end{array}$ & $\begin{array}{l}\text { To analyze the effect } \\
\text { of the Halliwick } \\
\text { method on physical } \\
\text { fitness }\end{array}$ & $\begin{array}{l}\text { PPS } \\
\mathrm{n}=1(\mathrm{~F}) \\
35 \text { years }\end{array}$ & $\begin{array}{l}\text { AT ( } 10 \text { min warm-up; } 70 \text { min } \\
\text { hydrokinesiotherapy program } \\
\text { using Halliwick method - mental } \\
\text { adjustment, release, vertical } \\
\text { rotation, lateral rotation, } \\
\text { combined rotation, flotation, } \\
\text { balance, weathering turbulence, } \\
\text { basic movement and fundamental } \\
\text { movements; } 10 \text { min cool down) }\end{array}$ & $\begin{array}{l}90 \mathrm{~min}, 5 \text { days/week } \\
\text { for } 4 \text { months }\end{array}$ & NR & $\begin{array}{l}\text { Pre/post intervention ( } \Delta \% \text { percentage } \\
\text { pre-post change) } \\
\text { - Senior Fitness Test: } \\
\text { Arm curl test: right arm } \uparrow 361.5 \Delta \% \text {; left } \\
\text { arm } \uparrow 360 \Delta \% \\
\text { Back scratch test: right shoulder } \uparrow 100 \Delta \% \text {; } \\
\text { left shoulder } \uparrow 125 \Delta \%\end{array}$ & $\begin{array}{l}\text { Case } \\
\text { study }\end{array}$ \\
\hline
\end{tabular}




\begin{tabular}{|c|c|c|c|c|c|c|c|}
\hline $\begin{array}{l}\text { Medeiros et al. } \\
\text { (2018) [43] }\end{array}$ & $\begin{array}{l}\text { To investigate the } \\
\text { effects of a AT on } \\
\text { biochemical } \\
\text { parameters, quality of } \\
\text { life and functional } \\
\text { physical capacity }\end{array}$ & $\begin{array}{l}\text { PPS } \\
\mathrm{n}=2(\mathrm{~F}) \\
53 \text { years } \\
\text { AT: } \mathrm{n}=1 \\
\text { No PA: } \mathrm{n}=1 \\
57 \text { years }\end{array}$ & AT (swimming program) & $\begin{array}{l}60 \mathrm{~min}, 2 \text { days/week } \\
\text { for } 21 \text { sessions }\end{array}$ & $27^{\circ} \pm 1 \mathrm{C}$ & $\begin{array}{l}\text { Pre/post intervention } \\
\text { - Beck Anxiety Inventory: AT: } \downarrow 42 \% \text {; no } \\
\text { PA: } \uparrow 13 \% \\
\text { - BMI: AT: } \downarrow 7 \% \text {; no PA: } \uparrow 9 \% \\
\text { - FM: AT: } \downarrow 5 \% \text {; no PA: } \uparrow 10 \% \\
\text { - Flexibility test: AT: } \uparrow 23 \% \text {; no PA: no } \\
\text { change } \\
\text { - Hip circumference: AT: } \downarrow 3 \% \text {; no PA: } \uparrow 5 \% \\
\text { - Muscle strength: AT: } \uparrow 10 \% \text {; no PA: no } \\
\text { change } \\
\text { - SF36: } \\
\text { Role limitations due to mental health: AT: } \\
\uparrow 11 \% \text {; no PA: no change } \\
\text { General health: AT: } \uparrow 13 \% ; \text { no PA: no change } \\
\text { - Waist circumference: AT: } \downarrow 4 \% \text {; no PA: } \\
\uparrow 1 \%\end{array}$ & $\begin{array}{l}\text { Case } \\
\text { study }\end{array}$ \\
\hline $\begin{array}{l}\text { Salem et al. } \\
(2010) \text { [45] }\end{array}$ & $\begin{array}{l}\text { To describe an AT } \\
\text { emphasizing } \\
\text { functional movement } \\
\text { to evaluate changes in } \\
\text { motor function during } \\
\text { land-based activities }\end{array}$ & $\begin{array}{l}\text { SMA } \\
\text { Type: III } \\
\mathrm{n}=1(\mathrm{~F}) \\
3 \text { years }\end{array}$ & $\begin{array}{l}\text { AT (breathing exercises, } \\
\text { flexibility, walking activities, } \\
\text { exercised for last } 5 \text { min with the } \\
\text { water between the mid-thigh } \\
\text { level and the mid-leg level to } \\
\text { allow transition of the activities } \\
\text { to the land) }\end{array}$ & $\begin{array}{l}45 \text { min, } 2 \text { days/week, } \\
14 \text { weeks }\end{array}$ & $33^{\circ} \mathrm{C}$ & $\begin{array}{l}\text { Pre/post intervention } \\
\text { - Gait speed: } \uparrow 0.25 \mathrm{~m} \\
\text { - Gross Motor Function Measure- } 88: \uparrow 11 \% \\
\text { - MMT: } \uparrow \text { lower limb muscles (exception of } \\
\text { the right hamstring and bilateral dorsiflexor } \\
\text { strength which remained the same) } \\
\text { - Peabody Developmental Motor Scales-2 } 2^{\text {nd }} \\
\text { Edition: } \uparrow 8 \text { pts } \\
\text { - Single-limb support time: } \uparrow 4.5 \% \text { of the gait } \\
\text { cycle } \\
\text { - Stride length: } \uparrow 14 \mathrm{~cm} \\
\text { - Stride time: } \downarrow 0.21 \mathrm{~s}\end{array}$ & $\begin{array}{l}\text { Case } \\
\text { study }\end{array}$ \\
\hline $\begin{array}{l}\text { Silva et al. } \\
\text { (2010) [50] }\end{array}$ & $\begin{array}{l}\text { To describe the effects } \\
\text { of the AT in } \\
\text { wheelchair patients } \\
\text { with PPS, in view of } \\
\text { trunk balance, pain, } \\
\text { and consequent } \\
\text { improvement of the } \\
\text { activities of daily life }\end{array}$ & $\begin{array}{l}\text { PPS } \\
\mathrm{n}=2(\mathrm{~F}) \\
\text { Case 1:44 years } \\
\text { (F) } \\
\text { Case 2:49 years } \\
\text { (F) }\end{array}$ & $\begin{array}{l}\text { AT (active exercises focused on } \\
\text { forward, lateral and back trunk } \\
\text { muscles, stretching, relaxation) }\end{array}$ & $\begin{array}{l}45 \mathrm{~min}, 2 \text { days/week } \\
\text { for } 24 \text { weeks }\end{array}$ & $33^{\circ} \mathrm{C}$ & $\begin{array}{l}\text { Pre/post intervention } \\
\text { - Barthel Index: Case } 1: \uparrow 27.7 \% \text {; Case } 2: \text { no } \\
\text { change } \\
\text { - BBS: Case } 1: \uparrow 21.5 \% \text {; Case } 2: \uparrow 5.4 \% \\
\text { - MMT: Case } 1: \uparrow \text { strength of trunk } \\
\text { extensors; Case } 2: \uparrow \text { strength of trunk } \\
\text { extensors and rotators } \\
\text { - VAS - pain scale: Case } 1: \downarrow 100 \% \text {; Case } 2 \text { : } \\
\downarrow 38.6 \%\end{array}$ & $\begin{array}{l}\text { Case } \\
\text { study }\end{array}$ \\
\hline
\end{tabular}


Table 4

Study Characteristics - Research on Other NMDs

\begin{tabular}{|c|c|c|c|c|c|c|c|}
\hline Reference & Study aim & Sample & Intervention program & Exercise parameters & $\begin{array}{l}\text { Water tem- } \\
\text { perature }\end{array}$ & Measuring instrument and outcome & $\begin{array}{l}\text { Study } \\
\text { design }\end{array}$ \\
\hline $\begin{array}{l}\text { Willen et al. } \\
\text { (2001) [19] }\end{array}$ & $\begin{array}{l}\text { To evaluate the } \\
\text { specific effects of } \\
\text { general dynamic } \\
\text { water exercise in } \\
\text { individuals with PPS }\end{array}$ & $\begin{array}{l}\text { PPS } \\
\mathrm{n}=28 \\
\text { EG: } 7(\mathrm{M}), 8(\mathrm{~F}) \\
\text { Mean: } 51 \text { years } \\
\text { CG: } 8(\mathrm{M}), 5(\mathrm{~F}) \\
\text { Mean: } 49 \text { years }\end{array}$ & $\begin{array}{l}\text { AT (aquatic exercises during } \\
\text { standing, walking, bicycling, } \\
\text { stretching) }\end{array}$ & $\begin{array}{l}40 \text { min, } 2 \text { days/week, } \\
8 \text { months }\end{array}$ & $+33^{\circ} \mathrm{C}$ & $\begin{array}{l}\text { Pre/post intervention } \\
-30 \text {-meter walking - no change } \\
\text { - BBS - no change } \\
\text { - Muscle strength (dynamometer) - no } \\
\text { change } \\
\text { - Peak } \mathrm{VO}_{2}: \mathrm{EG}: \downarrow 1.2 \pm 4.0 \mathrm{~mL} / \mathrm{kg} / \mathrm{min} \text {; } \\
\text { CG: } \uparrow 1.0 \pm 3.4 \mathrm{~mL} / \mathrm{kg} / \mathrm{min} \\
\text { - Physical Activity Scale for the Elderly: } \\
\text { EG: } \downarrow 4 \text {; CG: } \downarrow 12 \\
\text { - VAS - pain: EG: } \downarrow 15 \text { pts; CG: } \uparrow 4 \text { pts }\end{array}$ & $\begin{array}{l}\text { Quasi- } \\
\text { experi- } \\
\text { mental }\end{array}$ \\
\hline \multicolumn{8}{|c|}{ Peripheral Nervous System Neuropathies } \\
\hline $\begin{array}{l}\text { Leite et al. } \\
\text { (2010) [40] }\end{array}$ & $\begin{array}{l}\text { To analyze the effects } \\
\text { of AT associated to } \\
\text { kinesiotherapy on } \\
\text { land }\end{array}$ & $\begin{array}{l}\text { ChMTD } \\
\mathrm{n}=1(\mathrm{~F}) \\
49 \text { years }\end{array}$ & $\begin{array}{l}\text { AT (muscle strengthening, waist } \\
\text { dissociation, balance exercises, } \\
\text { jogging) + kinesiotherapy on land } \\
\text { (activity circuit, squat on the } \\
\text { parallel bars, kneeling to } \\
\text { standing) }\end{array}$ & $\begin{array}{l}60 \mathrm{~min}, 2 \text { days/week } \\
\text { for } 21 \text { sessions }\end{array}$ & $32-34^{\circ} \mathrm{C}$ & $\begin{array}{l}\text { Pre/post intervention } \\
\text { - } 6 \mathrm{MWT}: \uparrow 15.50 \mathrm{~m} \\
\text { - BBS: } \uparrow 6 \% \\
\text { - Gait speed: } \uparrow 0.11 \mathrm{~s} \\
\text { - SF36: } \\
\text { Physical function: } \uparrow 53.84 \% \\
\text { Role limitations due to physical health: } \uparrow 50 \% \\
\text { Role limitations due to mental health: } \\
\uparrow 4.16 \% \\
\text { Energy/fatigue: no change } \\
\text { Emotional well-being: no change } \\
\text { Social functioning: no change } \\
\text { Pain: no change } \\
\text { General health: } \uparrow 12.19 \% \\
\text { - TUG: } \uparrow 0.11 \mathrm{~s} \\
\text { - Up and down stairs: } \downarrow 0.06 \mathrm{~s} ; \downarrow 0.14 \mathrm{~s}\end{array}$ & $\begin{array}{l}\text { Case } \\
\text { study }\end{array}$ \\
\hline
\end{tabular}

- TUG: $\uparrow 0.11 \mathrm{~s}$ 


\begin{tabular}{|c|c|c|c|c|c|c|c|}
\hline $\begin{array}{l}\text { Mayer et al. } \\
(2020)[42]\end{array}$ & $\begin{array}{l}\text { To describe the effects } \\
\text { of land-based and AT }\end{array}$ & $\begin{array}{l}\text { Miller Fisher } \\
\text { syndrome } \\
\mathrm{n}=1(\mathrm{M}) \\
57 \text { years }\end{array}$ & $\begin{array}{l}\text { AT (postural control, righting } \\
\text { reactions, body awareness, gait } \\
\text { training, lower extremity, general } \\
\text { endurance, strength } \\
\text { exercises) + PT (balance training, } \\
\text { transfers and gait training, } \\
\text { stability during standing) }\end{array}$ & $\begin{array}{l}\text { AT }(60 \mathrm{~min}, 2 \\
\text { days/week for } 7 \\
\text { weeks })+ \text { PT }(60 \mathrm{~min} \text {, } \\
2 \text { days/week for } 7 \\
\text { weeks) }\end{array}$ & NR & $\begin{array}{l}\text { Pre/post intervention } \\
\text { - 6MWT: } 47.9 \mathrm{~m} \\
\text { - 10MWT: Self-Selected velocity } \uparrow 0.05 \mathrm{~m} / \mathrm{s} \text {; } \\
\text { Fast Velocity } \uparrow 0.21 \mathrm{~m} / \mathrm{s} \\
\text { - BBS: no change } \\
\text { - SF36: } \\
\text { Physical function: } \uparrow 5 \% \\
\text { Role limitations due to physical health: } \\
\uparrow 25 \% \\
\text { Role limitations due to mental health: } \\
\uparrow 100 \% \\
\text { Energy/fatigue } \uparrow 5 \% \\
\text { Emotional well-being: } \uparrow 40 \% \\
\text { Social functioning } \uparrow 12.5 \% \\
\text { Pain: no change } \\
\text { General health } \downarrow 10 \% \\
\text { - TUG: } \downarrow 26.81 \mathrm{~s}\end{array}$ & $\begin{array}{l}\text { Case } \\
\text { study }\end{array}$ \\
\hline $\begin{array}{l}\text { Zivi et al. } \\
\text { (2017) [21] }\end{array}$ & $\begin{array}{l}\text { To compare the effects } \\
\text { of AT in the context of } \\
\text { a land-based } \\
\text { rehabilitative program } \\
\text { and the land-based } \\
\text { rehabilitation alone on } \\
\text { balance and gait }\end{array}$ & $\begin{array}{l}\text { Peripheral } \\
\text { neuropathy } \\
\mathrm{n}=40 \\
\text { EG: } 11(\mathrm{M}), 10(\mathrm{~F}) \\
66.3(13.0) \text { years } \\
\text { CG: } 8(\mathrm{M}), 11(\mathrm{~F}) \\
71.8(7.7) \text { years }\end{array}$ & $\begin{array}{l}\text { EG: 1. AT (relaxation and breath } \\
\text { control; balance and posture } \\
\text { control exercises; gait } \\
\text { exercises) }+2 \text {. conventional } \\
\text { one-to-one training with a } \\
\text { physical therapist }+3 \text {. devices } \\
\text { training with the supervision of a } \\
\text { physical therapist (treadmill, } \\
\text { cycloergometer, cyclette, } \\
\text { stabilometric platform) }+4 \text {. } \\
\text { Occupational Therapy } \\
\text { CG: } 1 \text {. conventional one-to-one } \\
\text { training with a physical } \\
\text { therapist + 2. devices training } \\
\text { with the supervision of a physical } \\
\text { therapist (treadmill, } \\
\text { cycloergometer, cyclette, } \\
\text { stabilometric platform)+3. } \\
\text { Occupational Therapy }\end{array}$ & $\begin{array}{l}\text { weeks } \\
\text { EG: } 1.60 \mathrm{~min}, 3 \\
\text { days/week }+2.60 \mathrm{~min} . \\
2 \text { days/week } \\
+3.60 \mathrm{~min}, 6 \\
\text { days/week }+4.60 \mathrm{~min} \text {, } \\
5 \text { days/week } \\
\text { CG: } 1.60 \mathrm{~min}, 5 \\
\text { days/week }+2.60 \mathrm{~min} \text {, } \\
6 \text { days/week } \\
+3.60 \text { min, } 5 \\
\text { days/week }\end{array}$ & $32^{\circ} \mathrm{C}$ & $\begin{array}{l}\text { Pre/post intervention (delta) } \\
\text { - BBS: EG: } \uparrow 11.1 \pm 4.9 \text {; CG: } \uparrow 11.4 \pm 6.9 \\
\text { - Conley Scale: EG: } \downarrow 1.5 \pm 1.5 \text {; CG: } \\
\downarrow 0.9 \pm 1.4 \\
\text { - Dynamic Gait Index: EG: } \uparrow 5.8 \pm 2.1 \text {; CG: } \\
\uparrow 3.9 \pm 3.2 \\
\text { - Functional Ambulation Classification: EG: } \\
\uparrow 0.9 \pm 0.6 \text {; CG: } \uparrow 1.4 \pm 0.7 \\
\text { - Functional Independence Measure: EG: } \\
\uparrow 25.8 \pm 8.2 \text {; CG: } \uparrow 26.1 \pm 11.5 \\
\text { - Medical Research Council Scale score } \\
\text { Hip flexors: EG: } \uparrow 0.5 \pm 0.5 \text {; CG: } \uparrow 0.9 \pm 0.5 \\
\text { Hip extensors: EG: } \uparrow 0.8 \pm 0.4 \text {; CG: } \\
\uparrow 0.8 \pm 0.5 \\
\text { Ankle flexors EG: } \uparrow 0.7 \pm 0.7 \text {; CG: } \\
\uparrow 0.9 \pm 0.7 \\
\text { Ankle extensors EG: } \uparrow 0.6 \pm 0.7 \text {; CG: } \\
\uparrow 0.9 \pm 0.8 \\
\text { - Neuropathic Pain Scale: EG: } \downarrow 0.7 \pm 0.7 \text {; } \\
\text { CG: } \downarrow 0.5 \pm 1.8 \\
\text { - Overall Neuropathy Limitations Scale: } \\
\text { EG: } \downarrow 0.2 \pm 1.2 \text {; CG: } \downarrow 0.8 \pm 0.9\end{array}$ & RCT \\
\hline
\end{tabular}


Table 4

Study Characteristics - Research on Other NMDs

\begin{tabular}{|c|c|c|c|c|c|c|c|}
\hline Reference & Study aim & Sample & Intervention program & Exercise parameters & $\begin{array}{l}\text { Water tem- } \\
\text { perature }\end{array}$ & Measuring instrument and outcome & $\begin{array}{l}\text { Study } \\
\text { design }\end{array}$ \\
\hline \multicolumn{8}{|c|}{ Mixed NMD } \\
\hline $\begin{array}{l}\text { Huguet- } \\
\text { Rodríguez et } \\
\text { al. (2020) [54] }\end{array}$ & $\begin{array}{l}\text { To evaluate } \\
\text { respiratory changes } \\
\text { and functional } \\
\text { outcomes in children } \\
\text { with NMD attending } \\
\text { an AT program }\end{array}$ & $\begin{array}{l}\text { DMD }(\mathrm{n}=2), \\
\text { congenital } \\
\text { myopathy }(\mathrm{n}=1), \\
\text { non-hereditary } \\
\text { neuromuscular } \\
\text { disease }(\mathrm{n}=1), \\
\text { Steinert muscular } \\
\text { dystrophy }(\mathrm{n}=1) \text {, } \\
\text { SMA }(\mathrm{n}=4) \\
\text { ChMTD }(\mathrm{n}=2) \\
6 \mathrm{M} / 5 \mathrm{~F} \\
8.36(4.4) \text { years }\end{array}$ & $\begin{array}{l}\text { AT (balance control, } \\
\text { proprioception, double tasking, } \\
\text { coordination, dissociation of } \\
\text { shoulder and pelvic girdles, } \\
\text { flexibility, maintenance of range } \\
\text { of motion) }\end{array}$ & $\begin{array}{l}45-60 \min , 1 \\
\text { day/week for } 10 \\
\text { weeks }\end{array}$ & NR & $\begin{array}{l}\text { Pre/post intervention } \\
\text { - Forced expiratory volume in one second: } \\
\uparrow 0.12 \mathrm{~L} \\
\text { - Inspired volume: significant } \uparrow \text { in } \\
\text { children }>11 \text { years } \\
-\mathrm{O}_{2} \text { Sat: } \uparrow \text { in children }>11 \text { years* } \\
\text { - PEDI: care: } \uparrow 3.37 \text { pts; mobility: } \uparrow 3 \text { pts; } \\
\text { social: } \uparrow 1.45 \text { pts } \\
\text { - Pediatric quality of life inventory: } \\
\text { Disease: } \uparrow 0.99 \text { pts; communication: } \uparrow 4.17 \\
\text { pts; family: } \uparrow 2.86 \text { pts } \\
\text { - Strength of the respiratory muscles: } \\
\text { MEP } \uparrow 4.7 \text { pts; MIP } \downarrow 8.7 \text { pts } \\
- \text { Volume of inhaled air }: \uparrow 18 \text { pts } \\
\text { - Water Orientation Test Alyn } 1: \uparrow 3.37 \text { pts }\end{array}$ & $\begin{array}{l}\text { Quasi- } \\
\text { experi- } \\
\text { mental }\end{array}$ \\
\hline
\end{tabular}

$\uparrow$ - increase of the parameter; $\downarrow$ - decrease of the parameter; 6MWT - 6-Minute Walk Test; 10MWT - 10 Meter Walk Test; ALS - Amyotrophic Lateral Sclerosis; AT - aquatic therapy; BBS Berg Balance Scale; BMI - Body Mass Index; CG - control group; ChMTD - Charcot-Marie-Tooth Disease; DMD - Duchenne Muscular Dystrophy; EG - experimental group; F - female; FM - fat mass; M - male; MEP - Maximum Expiratory Pressure; MIP - Maximum Inspiratory Pressure; MMT- Manual Muscular Test; NMD - Neuromuscular Disorders; NR - not reported; PA physical activity; Peak $\mathrm{VO}_{2}$ - peak oxygen consumption; PEDI - Pediatric Evaluation of Disability Inventory; PPS - Post-polio syndrome; PT - physiotherapy; RCT - randomized clinical trial; SF-36 - Short Form (36) Health Survey; SMA - Spinal Muscular Atrophy; TUG - Timed Up \& Go Test; VAS - Visual Analog Scale. 
NMD studies have often used small group. One reason for this is that clinicians did not prescribe exercise for this group in the past, as they believed physical activity might cause muscle damage and overuse. However, there is growing evidence suggesting that aerobic exercise may have positive effects on persons with NMD [60]. Due to its low-impact nature, the aquatic environment creates a movement opportunity with a low risk of injury and falling, which is ideal for people with weaker muscles. Immersion in the water also elicits physiological responses from the cardiac and respiratory system, including an increase in cardiac output and an increase in the work of breathing, resulting in changes in respiratory dynamics [18]. Our present review yielded ambiguous results regarding respiratory measures in muscular dystrophies; this again, could be a consequence of the noted differences in sample sizes and AT intervention parameters. None of the studies with persons with SMA and ALS investigated any changes in respiratory system, despite the severity of the respiratory progression in these diseases [14, 61]. Three studies used heart rate monitors to control the intensity of the sessions and only one study performed proper HR monitoring during every session to ensure training at a safe level [36]. Although immersion is not contraindicated for cardiopathies [62], such as individuals with Duchenne and Becker $\mathrm{MD}$, these individuals should be screened prior to immersion to ensure appropriate safety precautions are implemented [6]. Nevertheless, none of the studies from our review reported screening before the intervention.

Aquatic therapy for general rehabilitation purposes and special populations is commonly performed in warm (above $30^{\circ} \mathrm{C}$ ), shallow (chest-deep) water and may involve a variety of exercise modalities including aerobic, stretching/range of motion, resistance, and stability training [15]. Only five studies reported the implementation of specific methods for AT (Halliwick, WETSwim and Bad Ragaz) in the intervention programs for individuals with NMDs [39, 41, 44, $51,55]$. There are no recommendations on how to set fundamental parameters when scheduling aquatic sessions for persons with NMD, such as duration of the intervention, frequency, type of exercises, water temperature, level of immersion.

Our findings highlight considerable differences between studies in terms of duration of the intervention, which ranged from five weeks to two years. As NMDs are progressive diseases, longitudinal studies could detect developments or changes in patient populations at both the group and individual level [63]. The most common frequency of AT sessions was two times a week, which allows other therapies to be incorporated in the treatment.

Research on neurological diseases suggest that in persons with stroke [25] and Parkinson's [64], AT may be the most effective approach to addressing activity limitation when it is complemented with land-based therapy. Our review showed that all studies conducted on persons with PNS, five studies with myopathy [20, 33, 52, 53, 55], and one with CNS [51] also incorporated aquatic and land therapy. Hind et al. [20] report lower motor function loss in an experimental group treated with aquatic and land therapy, compared to a group which received only land therapy.

Regarding the measured outcomes, most were therapeutically oriented, focusing on the functional performance and/or physiological domain, which is similar in patients with other neurologic conditions [65]. The majority of studies showed improvement or no change after AT intervention. It has to be noted that in the case of progressive diseases, a lack of decrease in outcomes is regarded as a positive therapeutic goal for maintaining function.

Some specific aspects were observed with regard to particular types of NMD. In individuals with myopathy, the physiological outcomes tended to concentrated on the loss of respiratory function, which is commonly observed in this condition. Our review identified some inconsistency in reported respiratory outcomes, which again, could be a consequence of differences in sample sizes and AT interventions. Moreover, Voos et al. [53] suggest that some persons with MD show respiratory function impairments according to spirometry, but good mouth and nose expiratory times during immersion; this may indicate that several compensatory strategies are available in the aquatic environment.

In the studies related to AT interventions for individuals with CNS diseases, the authors focused more on the effects of AT on muscular strength, activities of daily living and motor gross function. Interestingly, assessment of pain and quality of life was more addressed in PPS compared to persons with myopathy and/or PNS diseases. Three studies analyzing the implementation of AT in individuals with PNS reported improvement mainly in functional performance $[21,40,42]$, but the focus was on ambulation. The authors report that AT had positive effects on gait speed, distance, risk of falling and body balance. Surprisingly, only five of the twenty-eight studies 
considered aquatic skills as AT outcomes. Ferreira et al. [55] observed an improvement of aquatic skills but a decrease of motor function on land, measured by EK, after two years. This might indicate that, perhaps due to the properties of the water environment, AT is able to activate preserved muscles that are not strong enough to overcome the force of the gravity, thus reinforcing the recommendation of AT.

\section{Limitations}

The results should be interpreted with caution, because in order to cover as broad a spectrum of the literature as possible, no quality inclusion criteria were used. It is possible that some relevant studies were not included in the chosen search strategy. However, the risk of selection bias was limited by the use of the detailed search terms and screening performed by two independent reviewers. This review did not include broad grey literature search and studies in other languages than English, Portuguese and Spanish.

\section{CONCLUSIONS}

Considerable heterogeneity exists between and within AT interventions for individuals with NMD. The fundamental parameters and procedures of therapy should be standardized to allow a better interpretation of the benefits. Although our mapping illustrates the diverse nature of the targeted outcomes of interventions, it has to be noted that fatigue, which is one of the most relevant symptoms of NMDs was rarely analyzed. Additionally, AT in CNS diseases needs more investigation concerning respiratory system assessment.

The comprehensive outline of available literature given in this scoping review could serve as a starting point for high-level clinical studies on the effects of AT on persons with NMD and improve the definition of parameters and protocols for its consensus. Future research based on NMD populations should focus on aspects related to optimal intervention; these should include the duration, frequency and type of program (aquatic, land-based or combined), the exercise parameters (water temperature and depth, water immersion level, starting intensity, time of exercise or number of repetitions, equipment used) and validated outcome measures (regarding body structures and functions, activities and participation).

\section{ACKNOWLEDGMENTS}

This work was supported by the Ministry of Science and Higher Education in the year 2021 under Research Group no 4 at Jozef Pilsudski University of Physical Education in Warsaw "Physical activity and sports for people with special needs".

\section{DECLARATION OF INTEREST}

The authors have no conflicts of interest to report.

\section{REFERENCES}

[1] Dowling JJ, D Gonorazky H, Cohn RD, Campbell C. Treating pediatric neuromuscular disorders: The future is now. Am J Med Genet A. 2018;176(4):804-41.

[2] Gilbreath HR. Common neuromuscular disorders in Pediatrics. Physician Assist Clin. 2016;1:583-97.

[3] Bertorini TE. Neuromuscular Disorders. Philadelphia, USA: Saunders Elsevier; 2011.

[4] McDonald CM. Clinical approach to the diagnostic evaluation of hereditary and acquired neuromuscular diseases. Phys Med Rehabil Clin N Am. 2012;23(3):495-563.

[5] Benarroch L, Bonne G, Rivier F, Hamroun D. The 2021 version of the gene table of neuromuscular disorders (nuclear genome). Neuromuscul Disord. 2020;30(12):1008-48.

[6] Birnkrant DJ, Bushby K, Bann CM, Alman BA, Apkon $\mathrm{SD}$, Blackwell A, et al. Diagnosis and management of Duchenne muscular dystrophy, part 2: Respiratory, cardiac, bone health, and orthopaedic management. Lancet Neurol. 2018;17(4):347-61.

[7] Tawil R, Mah JK, Baker S, Wagner KR, Ryan MM. Clinical practice considerations in facioscapulohumeral muscular dystrophy Sydney, Australia, 21 September 2015. Neuromuscul Disord. 2016;26(7):462-71.

[8] Wong BL. Management of the child with weakness. Semin Pediatr Neurol. 2006;13(4):271-8.

[9] Mercuri E, Finkel RS, Muntoni F, Wirth B, Montes J, Main $\mathrm{M}$, et al. Diagnosis and management of spinal muscular atrophy: Part 1: Recommendations for diagnosis, rehabilitation, orthopedic and nutritional care. Neuromuscul Disord. 2018;28(2):103-15.

[10] Martini J, Hukuda ME, Caromano FA, Favero FM, Fu $\mathrm{C}$, Voos MC. The clinical relevance of timed motor performance in children with Duchenne muscular dystrophy. Physiother Theory Pract. 2015;31(3):173-81.

[11] Gayraud J, Ramonatxo M, Rivier F, Humberclaude V, Petrof B, Matecki S. Ventilatory parameters and maximal respiratory pressure changes with age in Duchenne muscular dystrophy patients. Pediatr Pulmonol. 2010;45(6): 552-9.

[12] Morse CI, Bostock EL, Twiss HM, Kapp LH, Orme P, Jacques MF. The cardiorespiratory response and physiological determinants of the assisted 6-minute handbike cycle test in adult males with muscular dystrophy. Muscle Nerve. 2018;58(3):427-33.

[13] Quinn L, Morgan D. From Disease to Health: Physical Therapy Health Promotion Practices for Secondary Prevention in Adult and Pediatric Neurologic Populations. J Neurol Phys Ther. 2017;41(Suppl 3):S46-S54. 
[14] Andersen PM, Abrahams S, Borasio GD, de Carvalho M, Chio A, Van Damme P, et al. EFNS guidelines on the clinical management of amyotrophic lateral sclerosis (MALS)-revised report of an EFNS task force. Eur J Neurol. 2012;19(3):360-75.

[15] Plecash AR, Leavitt BR. Aquatherapy for neurodegenerative disorders. J Huntingtons Dis. 2014;3(1):5-11.

[16] Brunton LK, Bartlett DJ. Description of exercise participation of adolescents with cerebral palsy across a 4-year period. Pediatr Phys Ther. 2010;22(2):180-7.

[17] Hurvitz EA, Leonard C, Ayyangar R, Nelson VS. Complementary and alternative medicine use in families of children with cerebral palsy. Dev Med Child Neurol. 2003;45(6):364-70.

[18] Becker BE. Aquatic therapy: Scientific foundations and clinical rehabilitation applications. PM R. 2009;1(9): 859-72.

[19] Willén C, Sunnerhagen KS, Grimby G. Dynamic water exercise in individuals with late poliomyelitis. Archives of Physical Medicine and Rehabilitation. 2001;82(1):66-72.

[20] Hind D, Parkin J, Whitworth V, Rex S, Young T, Hampson L, et al. Aquatic therapy for boys with Duchenne muscular dystrophy (DMD): An external pilot randomised controlled trial. Pilot Feasibility Stud. 2017;3:16.

[21] Zivi I, Maffia S, Ferrari V, Zarucchi A, Molatore K, Maestri $\mathrm{R}$, et al. Effectiveness of aquatic versus land physiotherapy in the treatment of peripheral neuropathies: A randomized controlled trial. Clinical Rehabilitation. 2018;32(5): 663-70.

[22] de Lima AAR, Cordeiro L. Aquatic physical therapy in individuals with muscular dystrophy: Systematic scoping review. Fisioter Pesqui. 2020;27:100-11.

[23] Roostaei M, Baharlouei H, Azadi H, Fragala-Pinkham MA. Effects of Aquatic Intervention on Gross Motor Skills in Children with Cerebral Palsy: A Systematic Review. Phys Occup Ther Pediatr. 2017;37(5):496-515.

[24] Marinho-Buzelli AR, Bonnyman AM, Verrier MC. The effects of aquatic therapy on mobility of individuals with neurological diseases: A systematic review. Clinical Rehabilitation. 2015;29(8):741-51.

[25] Moritz TA, Snowdon DA, Peiris CL. Combining aquatic physiotherapy with usual care physiotherapy for people with neurological conditions: A systematic review. Physiotherapy Research International. 2020;25(1):N.PAG-N.PAG.

[26] Peters MDJ, Godfrey C, McInerney P, Munn Z, Tricco AC, Khalil H. Chapter 11: Scoping Reviews (2020 version). 2020. In: JBI Manual for Evidence Synthesis [Internet].

[27] Grant MJ, Booth A. A typology of reviews: An analysis of 14 review types and associated methodologies. Health Info Libr J. 2009;26(2):91-108.

[28] Tricco AC, Lillie E, Zarin W, O'Brien KK, Colquhoun H, Levac D, et al. PRISMA Extension for Scoping Reviews (PRISMA-ScR): Checklist and Explanation. Ann Intern Med. 2018;169(7):467-73.

[29] Honório S, Batista M, Martins J. The influence of hydrotherapy on obesity prevention in individuals with Duchenne Muscular Dystrophy. J Phys Educ Sport. 2013;13:140-6.

[30] Honório S, Batista M, Paulo R, Mendes P, Santos J, Serrano $\mathrm{J}$, et al. Aquatic influence on mobility of a child with duchenne muscular dystrophy: Case study. Ponte: Int Sci Res J. 2016;72:337-50.

[31] Dimitrova EN, Božinovikj I, Ristovska S, Pejcikj AH, Kolevska A, Hasani M. The Role of Rehabilitation in the Management of Patients with Charcot-Marie-Tooth Dis- ease: Report of Two Cases. Open Access Maced J Med Sci. 2016;4(3):443-8.

[32] Strumse YA, Stanghelle JK, Utne L, Ahlvin P, Svendsby EK. Treatment of patients with postpolio syndrome in a warm climate. Disabil Rehabil. 2003;25(2):77-84.

[33] Adams MA, Chandler LS. Effects of physical therapy program on vital capacity of patients with muscular dystrophy. Physical Therapy. 1974;54:494-6.

[34] Albarello PM, Spalvieri DF. Effects of Aquatic Therapy in an individual with Post-Polio Syndrome. Rev Neurocienc. 2012;20:399-403.

[35] Atamturk H, Atamturk A. Therapeutic effects of aquatic exercises on a boy with Duchenne muscular dystrophy. J Exerc Rehabil. 2018;14(5):877-82.

[36] DiBiasio P, Primus KB, Drogos J, Husted C, Stephens H, Thorpe D. Case Report An Aquatic Exercise Program for an Adolescent With Limb Girdle Muscular Dystrophy. J Aquatic Phys Ther. 2015;23(1):3-13.

[37] Fachardo GA, Carvalho SCP, Melo-Vitorino DF. Hydrotherapy treatment in Duchenne Muscular Distropy: A case report. Rev Neurocienc. 2004;12:217-21.

[38] Israel VL. Aquatic Physical Therapy: The Aquatic Functional Assessment Scale (AFAS) in Muscular Dystrophy. J Aquatic Phys Ther. 2018;26:21-9.

[39] Johnson CR. Aquatic therapy for an ALS patient. Am J Occup Ther. 1988;42(2):115-20.

[40] Leite HR, Batista AC, Corrêa CL. Hydrotherapy associated to kinesiotherapy in a patientt with Charcot-Marie-Tooth disease: Case report. Ver Neurocienc. 2010;18:485-90.

[41] Martínez P, López J, Valenzuela A. Hydrokinesitherapy program using the Halliwick method on strength endurance and flexibility in a person with poliomyelitis sequelae. Nutricion Hospitalaria. 2015;31:1452-4.

[42] Mayer JE, McNamara CA, Mayer J. Miller Fisher syndrome and Guillain-Barré syndrome: Dual intervention rehabilitation of a complex patient case. Physiother Theory Pract. 2020:1-10.

[43] Medeiros MS, Motta J, Mariano S, Menguer L, Silva LA. Case study of individualized swimming program in poliomielite beach chain: Analysis of biochamical parameters, quality in life and functional physical capability. Revista Brasileira de Ciencias Do Esporte. 2018;40:94-9.

[44] Nunes GA, Sandri TB, Gold V, Sachelli T, Mazzitelli C. Influence of the Aquatic Physiotherapy in the Infant Stress in Patient with Duchenne Muscular Dystrophy. Revista Brasileira de Ciências Da Saúde. 2008;30:313-24.

[45] Salem Y, Gropack SJ. Aquatic therapy for a child with type III spinal muscular atrophy: A case report. Phys Occup Ther Pediatr. 2010;30(4):313-24.

[46] Sales I, Clebis NK, Stabille SR. Effects of physical exercises in swimming pool on lung function of a duchenne's muscular dystrphy patient. A care report. Arq Ciênc Saúde Unipar. 2004;8:67-72.

[47] Sanders ME, Torres LE. Aquatic exercise for muscular dystrophy: The story of Laura Sos, Castellón, Spain. ACSM's Health \& Fitness Journal. 2010;14(6):35-41.

[48] Santos CPA, Hengles RC, Cyrillo FN, Rocco FM, Braga DM. Aquatic physical therapy in the treatment of a child with merosin-deficient congenital muscular dystrophy: Case report. Acta Fisiatr. 2016;23:102-6.

[49] Silva KM, Braga DM, Hengles RC, Beas ARV, Rocco FM. The impact of aquatic therapy on the agility of a non-ambulatory patient with Duchenne muscular dystrophy. Acta Fisiatr. 2012;19:42-5. 
[50] Silva TM, Conceição ÉCG, Quadros AAJ, Cunha MCB, Oliveira ASB. Aquatic exercises for trunk balance improvement in Post-polio syndrome patients - two cases report. Rev Neurocienc. 2010;18:36-43.

[51] Cunha MC, Oliveira AS, Labronici RH, Gabbai AA. Spinal muscular atrophy type II (intermediary) and III (KugelbergWelander). Evolution of 50 patients with physiotherapy and hydrotherapy in a swimming pool. Arq Neuropsiquiatr. 1996;54(3):402-6.

[52] Ramos FAB, Ordonho MC, de Resende TCV, Lima CB, de Vasconcelos CR, Lins e Silva DA. Evaluation of the respiratory muscle force and the peak flow in patients with Duchenne muscular dystrophy exposed to non-invasive ventilation and hydrotherapy. Pulmão RJ. 2008;17:81-6.

[53] Voos MC, Goya PSA, de Freitas BL, Pires AMT, Favero FM, Caromano FA. Timed immersion expiration measures in patients with muscular dystrophies. Arch Physiother. 2020;10:4.

[54] Huguet-Rodríguez M, Arias-Buría JL, Huguet-Rodríguez B, Blanco-Barrero R, Braña-Sirgo D, Güeita-Rodríguez J. Impact of Aquatic Exercise on Respiratory Outcomes and Functional Activities in Children with Neuromuscular Disorders: Findings from an Open-Label and Prospective Preliminary Pilot Study. Brain Sci. 2020;10(7).

[55] Ferreira AVS, Goya PSA, Ferrari R, Durán M, Franzini $\mathrm{RV}$, Caromano FA, et al. Comparison of motor function in patients with Duchenne muscular dystrophy in physical therapy in and out of water: 2-year follow-up. Acta Fisiátrica. 2015;22:51-4.

[56] Caromano FA, Kuga LS, Passarella J, Sá CSC. Physiological effects of hidrotherapic sesión in children with Duchenne muscular distrophy. Fisioter Pesqui. 1998;5:49-55.
[57] Deenen JC, Horlings CG, Verschuuren JJ, Verbeek AL, van Engelen BG. The Epidemiology of Neuromuscular Disorders: A Comprehensive Overview of the Literature. J Neuromuscul Dis. 2015;2(1):73-85.

[58] Anziska Y, Inan S. Exercise in neuromuscular disease. Semin Neurol. 2014;34:542-56.

[59] Fowler WM. Role of physical activity and exercise training in neuromuscular diseases. American journal of physical medicine \& rehabilitation / Association of Academic Physiatrists. 2002;81(11 Suppl):S187-95.

[60] Voet NBM. Exercise in neuromuscular disorders: A promising intervention. Acta Myol. 2019;38(4):207-14.

[61] Finkel RS, Mercuri E, Meyer OH, Simonds AK, Schroth MK, Graham RJ, et al. Diagnosis and management of spinal muscular atrophy: Part 2: Pulmonary and acute care; medications, supplements and immunizations; other organ systems; and ethics. Neuromuscul Disord. 2018;28(3):197207.

[62] Meyer K, Leblanc MC. Aquatic therapies in patients with compromised left ventricular function and heart failure. Clin Invest Med. 2008;31(2):E90-7.

[63] Caruana EJ, Roman M, Hernández-Sánchez J, Solli P. Longitudinal studies. J Thorac Dis. 2015;7(11):E537-40.

[64] Palamara G, Gotti F, Maestri R, Bera R, Gargantini $\mathrm{R}$, Bossio F, et al. Land Plus Aquatic Therapy Versus Land-Based Rehabilitation Alone for the Treatment of Balance Dysfunction in Parkinson Disease: A Randomized Controlled Study With 6-Month Follow-Up. Archives of Physical Medicine and Rehabilitation. 2017;98(6):1077-85.

[65] Kubielska J, Kostka J, Miller E. Factors determining the functional abilities of patients after stroke. Adv Rehab. 2020;34(3):8-14. 